\title{
Relações entre a saúde mental da gestante e o apego materno-fetal
}

\author{
Patrícia Alvarenga \\ Maria Virgínia Machado Dazzani \\ Universidade Federal da Bahia \\ Cristiane Ajnamei dos Santos Alfaya \\ Universidade Federal do Recôncavo da Bahia \\ Eulina da Rocha Lordelo \\ Universidade Federal da Bahia \\ César Augusto Piccinini \\ Universidade Federal do Rio Grande do Sul
}

\begin{abstract}
Resumo
O estudo investigou as relações entre variáveis sociodemográficas, saúde mental da gestante e o apego materno-fetal no terceiro trimestre de gestação. Participaram do estudo 261 gestantes selecionadas através de amostragem por acessibilidade em quatro maternidades públicas. As gestantes responderam individualmente uma ficha de dados sociodemográficos, a Escala de Apego Materno-Fetal e o SRQ-20. A análise de regressão revelou que o número de filhos $(4 \%)$ e a saúde mental materna $(4,2 \%)$ explicaram parte da variância no apego materno-fetal. A escolaridade da mãe e do pai não esteve associada a essa variável. O modelo de regressão múltipla considerando os quatro fatores analisados, explicou $8,2 \%$ da variância nos escores de apego materno-fetal. Discutem-se as implicações dessas variáveis na formação do vínculo da mãe com o bebê durante a gestação.
\end{abstract}

Palavras-chave: saúde mental; apego materno-fetal; gestação.

\begin{abstract}
Relations between pregnant women's mental health and maternal-fetal attachment. This study investigated the relations among sociodemographic variables, pregnant women mental health, and maternal-fetal attachment in the third trimester of pregnancy. Participants were 261 pregnant women recruited from public maternity wards using a convenience sampling technique. Each pregnant woman completed a sociodemographic data form, the Maternal-Fetal Attachment Scale, and the SRQ-20. Regression analysis revealed that the number of children (4\%) and mothers' mental health (4.2\%) accounted for part of the variance in maternalfetal attachment scores. Father's and mother's schooling was not associated with this variable. Taking the four analyzed factors into account, a multiple regression model accounted for $8.2 \%$ of the variance in the maternal-fetal attachment scores. The implications of these variables for mother-infant bonding during pregnancy are discussed.
\end{abstract}

Keywords: mental health; maternal-fetal attachment; pregnancy.

$\mathrm{O}$ vínculo estabelecido pela mãe com o feto durante a gravidez tem sido considerado um importante preditor da qualidade da relação que a díade estabelecerá nos primeiros meses de vida (Shin, Park, \& Kim, 2006). Fatores fisiológicos, psicológicos e sociais, além do próprio comportamento do feto nos últimos meses da gestação, interferem na formação do vínculo, e a saúde mental da mulher tem importantes repercussões sobre esse aspecto específico da maternidade (DiPietro, 2010; Hart \& McMahon, 2006).

$\mathrm{O}$ conceito de apego materno-fetal tem sido utilizado na literatura para descrever a qualidade da relação da gestante com o feto. Ele foi definido por Cranley (1981) como o grau com o qual mulheres se engajam em comportamentos indicadores de afiliação e interação com o seu bebê durante a gravidez. $\mathrm{O}$ apego materno-fetal pode ser avaliado com base na frequência de comportamentos que expressam cuidado e comprometimento com o feto, como, por exemplo, alimentar-se bem, evitar substâncias nocivas, conversar com o bebê e acariciar a barriga (Salisbury, Law, LaGasse, \& Lester, 2003). Além de comportamentos que indicam envolvimento e preocupação com a criança, o apego materno-fetal também pode ser detectado através de expectativas, pensamentos e sentimentos da gestante, tais como a tentativa de imaginar o rosto e a personalidade do bebê, e o anseio por segurá-lo no colo ou amamentá-lo (Cranley, 
1981).

Segundo Shieh, Kravitz e Wang (2001) vários autores identificaram os atributos críticos do apego materno-fetal. Nesse sentido, os diferentes tipos de indicadores do apego materno-fetal podem ser agrupados em três dimensões que constituem esse construto: a cognitiva, a afetiva e a altruística. $\mathrm{O}$ apego cognitivo está relacionado ao desejo de conhecer, entender ou definir o feto. Corresponde à imagem mental do feto criada pela gestante, sua concepção dele como uma pessoa, e também à atribuição de características ou intenções ao feto. Essa dimensão do apego é observada quando a gestante é capaz de ver o feto como um ser autônomo e real, e tal processo é particularmente favorecido pela sensibilidade aos movimentos do feto.

O apego afetivo corresponde ao prazer associado aos pensamentos e fantasias que envolvem o bebê, ao contato indireto e interação com o feto. $\mathrm{O}$ fato de a gestante manifestar prazer e entusiasmo por comportamentos como acariciar a barriga e conversar com o bebê evidencia essa dimensão do apego materno-fetal.

Por fim, o apego altruístico se refere à iniciativa de proteger o feto e de preparar-se para a chegada do bebê. Essa dimensão está relacionada a comportamentos como evitar substâncias nocivas para a saúde e o desenvolvimento do feto, preocuparse com a sua saúde, evitar níveis excessivos de estresse físico e mental e fazer o acompanhamento pré-natal (Condon, 1985). Alguns estudos fornecem evidências de que os indicadores do apego materno-fetal aumentam ao longo da gestação, especialmente nos últimos meses, em função dos movimentos do feto (DiPietro, 2010; Shieh et al., 2001).

Com base nesse conceito, alguns estudos buscam identificar os preditores do apego materno-fetal. Características sociodemográficas da família como maior nível de instrução e menor número de filhos, parecem favorecer o apego maternofetal (Alhusen, 2008; Cannela, 2005). Além disso, evidenciase o papel do apoio social à gestante, de fatores fisiológicos característicos da própria gestação como a saúde da gestante e do bebê, e dos exames realizados no período pré-natal, com destaque para a ultrassonografia (Yarcheski et al., 2009). A literatura revela ainda o impacto da preocupação com a imagem corporal da mulher durante a gravidez e os movimentos do feto (Alhusen, 2008; Di Pietro, 2010; Huang, Wang, \& Chen, 2004). Por fim, variáveis psicológicas como níveis de ansiedade e depressão (Hart \& McMahon, 2006; Lindgren, 2001; Seymir, Sjögren, Welles-Nyström, \& Nissen, 2009), a autoestima e a personalidade materna têm sido associadas à formação do apego materno-fetal (Di Pietro, 2010; Yarcheski, Mahon, Yarcheski, Hanks, \& Cannella, 2009).

Estudos de revisão sistemática da literatura oferecem um panorama geral do impacto desses diferentes preditores. Yarcheski et al. (2009) realizaram um estudo meta-analítico com base em 183 pesquisas publicadas e não publicadas entre 1981 e 2006 sobre os preditores do apego materno-fetal, e determinaram o tamanho do efeito dessas variáveis. Os autores utilizaram o coeficiente de correlação $r$ de Pearson como indicador do tamanho do efeito, de acordo com os critérios estabelecidos por Cohen (1988), (citado por Yarcheski et al., 2009). Eles concluíram que o fator antecedente mais fortemente relacionado ao apego materno-fetal foi a idade gestacional, sendo que os estudos revisados revelaram que a medida que a gestação progride, o apego materno-fetal tende a aumentar. A realização de ultrassonografias durante a gestação também parece favorecer significativamente a formação do vínculo da gestante com o futuro bebê. Por outro lado, fatores como o planejamento e o risco na gravidez, segundo os autores, apresentaram efeitos pequenos ou triviais sobre a variável investigada.

Quanto às relações entre a saúde mental da gestante e o apego materno-fetal, os achados são controversos. A hipótese de que sintomas de transtornos mentais teriam impacto negativo sobre o apego materno-fetal não tem sido corroborada de forma consistente. Por exemplo, o estudo de Lindgren (2001), que avaliou a relação da depressão materna com o apego maternofetal em uma amostra de 252 gestantes no terceiro trimestre de gestação, revelou que a depressão explicou 3\% da variância no apego materno-fetal. Já o estudo de Hart e McMahon (2006), que avaliou indicadores de saúde mental e o apego maternofetal de 53 gestantes também no terceiro trimestre da gravidez, indicou que enquanto os escores de traço e estado de ansiedade das gestantes foram preditores consistentes do apego maternofetal, os escores de depressão materna não foram. $\mathrm{O}$ modelo de regressão que incluiu a idade da mãe, o traço e o estado de ansiedade explicou 29,2\% da variância no apego materno-fetal. A mesma tendência foi constatada no estudo de Seymir et al. (2009) que não encontrou diferença no escore total de apego materno-fetal ao comparar gestantes com e sem depressão. Contudo, os autores verificaram que as gestantes deprimidas sentiam-se menos envolvidas com o bebê e expressaram maior desconforto em relação a sua imagem corporal. O grupo de gestantes com depressão também apresentou maior contato físico e sensibilidade aos movimentos do feto do que o grupo de gestantes não deprimidas.

A revisão de Yarcheski et al. (2009) examinou 14 estudos que investigaram o impacto da ansiedade da gestante sobre o apego materno-fetal, e encontrou coeficientes de correlação que variaram de 0,17 a 0,21. Os mesmos autores analisaram 15 estudos sobre os efeitos da depressão sobre o apego maternofetal e também encontraram coeficientes baixos, que variaram de 0,17 a 0,19. Com uma proposta semelhante de revisão das publicações sobre o tema no período de 1981 a 2004, Cannela (2005) verificou que dos, 60 estudos envolvendo variáveis psicossociais como preditoras do apego materno-fetal, em apenas 23 foram encontradas correlações estatisticamente significativas. Entre essas 23 investigações, foram encontrados apenas dois estudos que verificaram correlações inversas da saúde mental da gestante com o apego materno fetal, um deles envolvendo ansiedade e o outro sobre depressão. Por outro lado, Alhusen (2008), que analisou 22 estudos publicados no período de 2000 a 2007 sobre fatores protetivos e fatores de risco para o apego materno-fetal, apontou para achados mais consistentes e conclusivos no que se refere à saúde mental materna. Segundo a autora, variáveis como a depressão, o abuso de substâncias e maiores níveis de ansiedade estariam associados a índices mais baixos de apego materno-fetal.

De qualquer modo, Alhusen (2008) e Cannella (2005) apontaram para as limitações dos estudos avaliados, 
principalmente com relação às características amostrais e às medidas de apego materno-fetal utilizadas. Com relação ao primeiro aspecto, Canella (2005) afirma que o tamanho das amostras e as técnicas de amostragem variaram muito entre os estudos, o que poderia explicar, pelo menos em parte, diferenças nos resultados. Quanto aos instrumentos, Doan, Cox e Zimerman (2003) afirmam que, de fato, existem vários questionamentos quanto à validade do principal instrumento utilizado para a avaliação do apego materno-fetal - a Escala de Apego Maternofetal de Cranley (1981). As principais críticas ao instrumento referem-se à falta de escores normativos provenientes da amostra que lhe deu origem e à ausência de um procedimento padronizado para o cálculo dos escores gerados pela escala. Além disso, dois pressupostos centrais enunciados por Cranley (1981) também são discutidos pela ausência de evidências a seu favor: a noção de que o apego materno-fetal se desenvolve em estágios e o pressuposto de que este atributo nem sempre se desenvolve normalmente. Segundo Doan et al. (2003), não está claro se as inconsistências encontradas na literatura sobre apego materno-fetal estão relacionadas a essas falhas, à conceituação das dimensões medidas pelo instrumento, ou ainda a interações da escala com fatores situacionais, ecológicos, demográficos e de personalidade. De qualquer modo, essa escala tem sido extensamente utilizada em estudos internacionais. No Brasil, os estudos que adotam esse instrumento são escassos.

Além dos estudos sobre os preditores do apego maternofetal, as pesquisas que investigam suas implicações para outras variáveis relacionadas à gestação e à qualidade da relação futura entre mãe e bebê evidenciam a relevância desse conceito para a psicologia do desenvolvimento. O estudo de Lindgren (2001), realizado com 252 gestantes adultas, investigou os efeitos diretos e indiretos da depressão (avaliada pelo CEDS - Center for Epidemiologic Studies Depression Scale) e do apego maternofetal (avaliada pela escala de Cranley) sobre a qualidade das práticas de cuidado com a saúde na gestação. Os resultados revelaram correlações significativas entre o nível educacional, o número de filhos, o apoio conjugal e as práticas de cuidado com a saúde. A depressão e o apego materno-fetal foram preditores de práticas positivas de cuidado com a saúde, mas em direções opostas. Enquanto a depressão correlacionou-se negativamente com tais práticas, o apego materno-fetal apresentou uma correlação positiva com esse fator. Já o estudo de Huang, Wang e Chen (2004) revelou que a decisão de amamentar o bebê ao peito esteve positivamente correlacionada com o apego materno-fetal.

Outros autores buscaram investigar a extensão com que o apego materno-fetal pode influenciar comportamentos maternos após o parto. Por exemplo, Shin, Park e Kim (2006) investigaram o impacto do apego materno-fetal sobre a sensibilidade materna após o nascimento do bebê, em 215 mulheres. Os resultados revelaram que quanto maior o escore de apego materno-fetal na gestação, maior o nível de sensibilidade materna relatado no período pós-parto. Contudo, esse estudo apresenta uma limitação metodológica por ter se baseado em dados retrospectivos, coletados no período entre uma e seis semanas após o parto, e, portanto, baseados em lembranças acerca do período gestacional.

Considerando a relevância do conceito de apego materno-fetal para a compreensão da relação mãe-bebê e do desenvolvimento infantil e os achados controversos a respeito do impacto da saúde mental materna sobre essa variável, o presente estudo investigou as relações entre variáveis sociodemográficas, saúde mental da gestante e apego materno-fetal no terceiro trimestre de gestação. A hipótese principal do estudo foi de que a maior frequência de indicadores de transtornos mentais maternos estaria relacionada a menores escores de apego materno-fetal.

\section{Método}

\section{Participantes}

Participaram do estudo 261 gestantes selecionadas através de amostragem por acessibilidade em quatro maternidades públicas de Salvador/Bahia. Todas as participantes encontravamse no terceiro trimestre de gestação no momento da coleta de dados. A escolaridade da gestante $(M=10,62 ; D P=2,62)$ e do pai do bebê $(M=10,52 ; D P=2,52)$ foi semelhante, e indica que a maior parte da amostra tinha ensino médio incompleto ou completo. Enquanto a maioria das gestantes não trabalhava fora (64\%), a maior parte dos pais estava empregada (92\%). Embora se trate de uma amostra proveniente de uma população de baixa renda, o número médio de filhos da gestante pode ser considerado baixo $(M=0,86 ; D P=1,13)$. Quanto à renda familiar, a média foi de aproximadamente um salário mínimo e meio $(M=\mathrm{R} \$ 828,82, D P=\mathrm{R} \$ 601,56)$. A maior parte das gestantes não apresentava problemas de saúde durante a gravidez $(85,4 \%)$. As gestantes que mencionaram problemas de saúde (14,6\%) destacaram: diabetes, asma, hipertensão, hipotensão e hipertireoidismo. O teste qui-quadrado não revelou associações estatisticamente significativas entre problemas de saúde e apego materno-fetal. A grande maioria das gestantes residia com o pai do bebê $(84,7 \%)$. A Tabela 1 apresenta detalhes dos dados sociodemográficos das participantes.

Ao serem questionadas sobre o planejamento da gravidez, $57,5 \%$ das gestantes responderam que a gravidez havia sido inesperada, e $42,5 \%$ disseram que a gravidez havia sido esperada. A grande maioria das gestantes sabia o sexo do bebê, sendo que $48,7 \%$ esperavam meninos e $36,8 \%$ esperavam meninas. Apenas 14,6\% das gestantes desconheciam o sexo do bebê. Pouco menos da metade da amostra foi constituída por mulheres que estavam em sua primeira gestação $(45,6 \%)$. Por fim, quando questionadas a respeito de complicações durante a gravidez, $72,4 \%$ das gestantes declararam não ter complicações, e 27,6\% afirmaram ter passado por complicações durante o período. Além dos problemas de saúde da gestante anteriores à gestação ou não relacionados a ela, também foram investigadas as complicações da gestação. Entre as participantes que mencionaram esse tipo de queixa, foram citadas as seguintes complicações: ameaça ou tentativa de aborto, hipertensão, eclampse e perda de líquido amniótico. Contudo, o teste qui-quadrado não revelou associações estatisticamente significativas entre complicações da gravidez e apego materno-fetal. A Tabela 2 apresenta informações sobre a gestação.

\section{Delineamento, procedimento e instrumentos}

Foi utilizado um delineamento correlacional (Robson, 
Tabela 1

Características sociodemográficas das participantes $(n=261)$

\begin{tabular}{|c|c|}
\hline Idade da gestante & $M=27,34(D P=5,52)$ \\
\hline Escolaridade da gestante (em anos) & $M=10,34(D P=2,62)$ \\
\hline Escolaridade do pai do bebê (em anos) & $M=10,24(D P=2,52)$ \\
\hline Número de fílhos & $M=0,86(D P=1,13)$ \\
\hline Tempo da união conjugal (em meses) & $M=60,37(D P=58,68)$ \\
\hline Número de moradores na casa & $M=2,42(D P=1,55)$ \\
\hline Renda familiar & $M=\mathrm{R} \$ 828,82(D P=\mathrm{R} \$ 601,56)$ \\
\hline \multicolumn{2}{|l|}{ Ocupacão da gestante } \\
\hline Trabalham fora & $35,6 \%$ \\
\hline Não trabalham fora & $64,4 \%$ \\
\hline \multicolumn{2}{|l|}{ Ocupação do pai do bebê } \\
\hline Trabalham fora & $92,0 \%$ \\
\hline Não trabalham fora & $8,0 \%$ \\
\hline \multicolumn{2}{|l|}{ Problemas de saúde da gestante } \\
\hline Sim & $14,6 \%$ \\
\hline Não & $85,4 \%$ \\
\hline \multicolumn{2}{|l|}{ A gestante vive com o pai do bebê? } \\
\hline Sim & $84,7 \%$ \\
\hline Não & $15,3 \%$ \\
\hline
\end{tabular}

Tabela 2

Condições da gestação $(n=261)$

\begin{tabular}{|c|c|}
\hline \multicolumn{2}{|l|}{ Planejamento da gravidez } \\
\hline Esperada & $42,5 \%$ \\
\hline Inesperada & $57,5 \%$ \\
\hline \multicolumn{2}{|l|}{ Sexo do bebê } \\
\hline Masculino & $48,7 \%$ \\
\hline Feminino & $36,8 \%$ \\
\hline Desconhecido & $14,6 \%$ \\
\hline \multicolumn{2}{|l|}{ Primeira gravidez } \\
\hline Sim & $45,6 \%$ \\
\hline Não & $54,4 \%$ \\
\hline \multicolumn{2}{|l|}{ Complicações na gravidez } \\
\hline Sim & $27,6 \%$ \\
\hline Não & $72,4 \%$ \\
\hline
\end{tabular}

1993) que envolveu, como variáveis preditoras, alguns fatores sociodemográficos e a saúde mental da gestante, e, como variável predita, o apego materno-fetal. Durante o terceiro trimestre da gravidez, as gestantes foram convidadas a participar da pesquisa e responderam individualmente, nos hospitais onde faziam o pré-natal, os seguintes instrumentos:

1) Ficha de Dados Sociodemográficos e Saúde da Gestante: Foi utilizada para investigar dados como idade da gestante, escolaridade, profissão, estado civil, existência de outros filhos, estado de saúde durante a gestação e data prevista para o nascimento do bebê.

2) Escala de apego materno-fetal (Cranley, 1981): Esta escala foi utilizada para avaliar o vínculo da gestante com o feto. $\mathrm{O}$ instrumento consta de 24 itens, divididos em cinco subescalas que representam diferentes aspectos da relação da mãe com o bebê. São exemplos de itens da escala: "Eu converso com meu bebê" e "Eu me imagino alimentando/amamentando o bebê". Para cada um dos itens, são oferecidas cinco opções de respostas em uma escala do tipo Likert, que variam de "claramente sim" a "claramente não". O índice de consistência interna (alpha de Cronbach) da escala original total é 0,85 ; e o da validação brasileira foi 0,63 (Feijó, 1999). A consistência interna e a validade de cada sub-escala isoladamente têm sido questionadas na literatura e (Doan et al., 2003), por esta razão não foram utilizadas no presente estudo.

3) SRQ-20 (Self-Report Questionnaire of Minor Psychiatric Disorders): Este instrumento foi desenvolvido por Harding et al. (1980, citado por Mari \& Williams, 1985). É uma escala composta de vinte e quatro itens, com o objetivo de detectar transtornos psiquiátricos menores através da investigação de sintomas nãopsicóticos (20 itens) e sintomas psicóticos (quatro itens). Para o presente estudo, de acordo com recomendações da literatura consultada (Mari \& Williams, 1986), foram utilizados somente os 20 itens destinados à avaliação dos sintomas não-psicóticos, tendo em vista a baixa fidedignidade da sub-escala de sintomas psicóticos. Investigou-se, portanto, a presença de sintomas de depressão, ansiedade e de algumas queixas psicossomáticas. $\mathrm{O}$ ponto de corte de oito pontos, isto é, oito respostas "sim" ou mais, foi utilizado para classificar cada mãe como "participante com suspeita de transtorno mental" ou como "participante sem suspeita de transtorno mental" (Mari \& Williams, 1986). Foi utilizada a versão brasileira do SRQ-20, validada por Mari e Williams (1986). O índice de consistência interna (alpha de Cronbach) da versão brasileira foi 0,86 (Gonçalves, Stein, \& Kapczinski, 2008).

\section{Resultados}

A avaliação da saúde mental das gestantes revelou que a amostra obteve, no SRQ-20, um escore médio de 8,01 (DP= $0,25)$. O escore total para cada participante poderia variar de um a 20 pontos e quanto mais alto o escore no SRQ-20, maior a frequência de indicadores de transtornos mentais. Além disso, é importante notar que o escore médio da amostra atingiu o ponto de corte do instrumento, que demarca as categorias com e sem suspeita de transtorno mental. Já o escore total de apego materno-fetal poderia variar de 24 a 120 pontos, e o escore médio foi de 91,21 $(D P=0,56)$. Embora esse valor esteja bem acima do ponto médio de escores da escala, não é possível fazer qualquer tipo de afirmação a respeito, uma vez que a escala não 
oferece escores normativos ou pontos de corte. Porém, o desvio padrão baixo associado tanto à média do escore de saúde mental quanto à média de apego materno-fetal indica que houve pouca variabilidade na amostra com relação a essas variáveis.

Algumas das características sociodemográficas investigadas foram correlacionadas com as variáveis saúde mental materna e apego materno-fetal. A Tabela 3 apresenta as correlações entre algumas variáveis sociodemográficas e os escores de saúde mental materna e apego materno-fetal.

Houve uma correlação negativa entre a escolaridade materna e a saúde mental da gestante $(r=-0,27 ; p=0,01)$, indicando que quanto menor a escolaridade materna maior o número de

\begin{tabular}{|c|c|c|}
\hline & Escore total no SRQ & $\begin{array}{l}\text { Escore na Escala de } \\
\text { apego materno-fetal }\end{array}$ \\
\hline Idade & 0,10 & $-0,07$ \\
\hline Escolaridade materna & $-0,27 * *$ & $0,12 *$ \\
\hline Número de filhos & $0,17 * *$ & $-0,20 * *$ \\
\hline Escolaridade do pai da criança & $-0,11$ & $0,14 *$ \\
\hline Tempo da união conjugal & 0,09 & $-0,08$ \\
\hline Número de moradores & 0,05 & $-0,09$ \\
\hline Renda familiar & $-0,21 * *$ & $-0,02$ \\
\hline
\end{tabular}

sintomas de transtornos psiquiátricos na gestante. Nessa mesma direção, foi encontrada também uma correlação negativa entre a renda familiar e a saúde mental da gestante $(r=-0,21 ; p=0,01)$, indicando que quanto menor a renda familiar maior o número de sintomas de transtornos psiquiátricos. Por fim, quanto maior o número de filhos maior o número de sintomas revelados pelos escores de saúde mental $(r=0,17 ; p=0,01)$.

A análise das correlações entre as variáveis sociodemográficas e o apego materno-fetal revelou uma correlação negativa entre o número de filhos e o apego materno fetal $(r=-0,20 ; p<$ $0,01)$, indicando que quanto maior o número de filhos menor o escore de apego da gestante com o feto durante a gestação. Além disso, a escolaridade materna e a escolaridade paterna apareceram positivamente correlacionadas ao escore na escala de apego materno fetal $(r=0,12 ; p<0,05$; e $r=0,14$; $p<0,05$, respectivamente), indicando que quanto maior a escolaridade maiores os escores de apego. Por fim, foi também encontrada uma correlação negativa entre o escore total da gestante no SRQ20 e o escore total na escala de apego materno-fetal $(r=-0,21 ; p$ $<0,01$ ), indicando que quanto maior o número de indicadores de transtornos psiquiátricos na gestante menor o apego da gestante com o feto durante a gestação.

As variáveis que apresentaram correlações significativas com o apego materno-fetal foram inseridas em uma análise de regressão linear múltipla. Os resultados revelaram que não houve indícios de que a escolaridade materna e a escolaridade paterna explicassem os escores de apego materno-fetal. Por outro lado, o número de filhos $(p=0,01)$ explicou $4 \%$ da variância no apego materno-fetal, desconsiderando a influência dos demais fatores. A saúde mental materna $(p=0,002)$ explicou $4,2 \%$ da variância nos escores de apego materno-fetal. O modelo de regressão múltipla $(F=6,34, p=0,001)$, considerando os quatro fatores analisados, explicou $8,2 \%$ da variância total nos escores de apego materno-fetal.

Por fim, foram também examinadas as relações entre algumas variáveis categóricas (planejamento da gravidez, primeira gravidez, viver com o pai e saúde mental da gestante) e o apego materno fetal, através do Teste $t$ para grupos independentes. A Tabela 4 apresenta os resultados dessas análises.

Os resultados revelaram que o escore médio de apego materno-fetal das gestantes que estavam em sua primeira gravidez $(M=92,72 ; D P=7,73)$ foi significativamente maior $(t=$ $2,90 ; p=0,04)$ do que o de gestantes que já haviam engravidado antes $(M=89,73 ; D P=8,75)$. Além disso, foi encontrada uma diferença significativa $(t=3,30 ; p=0,001)$, entre os escores de gestantes que apresentaram suspeita de transtorno mental e

Tabela 4

Média, desvio-padrão, valor de te nivel de significância dos escores de apego materno-fetal de acordo com o planejamento da gravidez, a ordem da gravidez, a presença do pai do bebê e a saúde mental da gestante $(n=261)$

\begin{tabular}{|c|c|c|c|}
\hline Variáveis & Média $(D P)$ & $t$ & $p$ \\
\hline \multicolumn{4}{|l|}{ Planejamento da gravidez } \\
\hline Gravidez esperada & $91,99(7,38)$ & $1,53 *$ & 0,12 \\
\hline Gravidez inesperada & $90,43(9,07)$ & & \\
\hline \multicolumn{4}{|l|}{ Primeira gravidez } \\
\hline Sim & $92,72(7,73)$ & 2,90 & 0,04 \\
\hline Não & $89,73(8,75)$ & & \\
\hline \multicolumn{4}{|l|}{ A gestante vive com o pai do bebê } \\
\hline Sim & $91,03(8,52)$ & 0,27 & 0,78 \\
\hline Não & $91,43(7,91)$ & & \\
\hline \multicolumn{4}{|l|}{ Saúde mental da gestante } \\
\hline Suspeita de transtorno mental & $89,65(8,16)$ & 330 & 0,001 \\
\hline Sem suspeita de transtorno mental & $93,07(8,40)$ & 3,30 & \\
\hline
\end{tabular}

$g l=259 ; * g l=256,63$ 
os escores de gestantes que não apresentavam suspeita desse tipo de transtorno, de acordo com o SRQ-20. As gestantes com suspeita de transtorno mental $(M=89,65 ; D P=8,16)$ tiveram escores significativamente menores do que as gestantes sem suspeita de transtorno mental $(M=93,07 ; D P=8,40)$. Por outro lado, não houve diferença significativa no apego materno fetal entre gestantes com gravidez esperada e gestantes com gravidez inesperada $(t=1,53 ; p=0,12)$, nem entre gestantes que viviam com o pai do bebê e gestantes que não viviam ou não com o pai do bebê $(t=0,27 ; p=0,78)$.

\section{Discussão}

A hipótese de que escores mais altos de indicadores de transtornos mentais estariam relacionados a menores escores de apego materno-fetal foi corroborada pelos resultados do presente estudo. Embora alguns estudos enfatizem a inconsistência dos achados a respeito das relações entre os dois fatores (Cannella, 2005; Yarcheski, 2009), as análises correlacionais, a análise de regressão e a comparação das médias dos escores entre mães com e sem suspeita de transtorno mental apontam consistentemente para o impacto negativo de sintomas psiquiátricos da gestante sobre o seu vínculo com o bebê no período pré-natal. Esta relação também foi encontrada por Alhusen (2008), que verificou que gestantes com mais sintomas de transtornos psiquiátricos obtiveram menores escores de apego materno fetal. Contudo, é importante salientar que, no presente estudo, as correlações foram baixas e que, na análise de regressão, a saúde mental da gestante explicou apenas uma parte pequena da variância no apego materno-fetal. Portanto, ao mesmo tempo em que esses achados corroboram a hipótese sobre a relação entre os dois fatores, os valores baixos obtidos nos testes indicam a influência de outras variáveis que, em futuras investigações, merecem ser articuladas à saúde mental e ao apego materno-fetal, em um modelo teórico menos linear e mais complexo (Shieh et al., 2001). Os pesquisadores têm apontado para a complexidade das relações que se estabelecem entre inúmeras variáveis de diferentes níveis durante a gestação, e a compreensão deste quadro requer um refinamento conceitual e metodológico que só poderá ser alcançado com o progresso das investigações nesta área.

De qualquer modo, os resultados apontam para a vulnerabilidade de mulheres com alta frequência de sintomas psiquiátricos no que se refere ao vínculo que está sendo estabelecido com o bebê. Segundo Seimyr et al. (2009), a depressão durante a gravidez pode ser um indicador de sentimentos ambivalentes em relação ao feto, à gestação e ao próprio corpo. Esses sentimentos ambivalentes fragilizariam o vínculo entre a mãe e o bebê e, consequentemente, tornariam menos frequentes os comportamentos indicadores de afeto e afiliação avaliados pela escala de apego materno-fetal. Além disso, o desânimo e os sentimentos de desesperança e desinteresse típicos dos quadros depressivos tendem a inibir, de modo geral, comportamentos e emoções que contribuem para a formação do vínculo (Lindgren, 2001).

Com relação aos sintomas de ansiedade, Hart e McMahon (2006) destacam a importância de distinguir a ansiedade específica relacionada ao bem-estar do bebê, da tendência geral a responder de forma ansiosa a situações do dia a dia. Segundo esses autores, a literatura indica que, enquanto a ansiedade específica em relação ao bebê tende a estar relacionada ao fortalecimento do apego materno-fetal, a tendência a um traço de ansiedade mais generalizado tende a prejudicar o vínculo. De qualquer modo, as razões que explicam os efeitos deletérios da ansiedade sobre o apego materno-fetal ainda não estão claras na literatura. Além dos sintomas de depressão e ansiedade, outros sintomas não-psicóticos avaliados pelo instrumento utilizado no presente estudo podem também produzir outras repercussões no apego materno-fetal. Porém, esses sintomas também não foram explorados de maneira específica pelo presente estudo nem pela literatura da área.

É importante destacar o caráter dinâmico da relação entre mãe e filho e sua complexidade, mesmo durante o período prénatal, pois esses aspectos do vínculo podem contribuir para a compreensão dos achados controversos nesta área. Por exemplo, Seimyr et al. (2009) encontraram uma relação entre depressão e a experiência da gestante em relação aos movimentos e ao contato físico com o feto. Ao contrário do que era esperado, gestantes com maiores escores de depressão também obtiveram maiores escores na sub-escala específica que avaliou o comportamento da mãe em relação aos movimentos do feto. Os autores levantaram algumas hipóteses para explicar esse fato. Uma delas seria a de que mães deprimidas e, talvez, ambivalentes em relação à gravidez, sentir-se-iam mais irritadas ao perceber os movimentos do feto e, portanto, teriam uma percepção mais acurada de sua atividade física, além do que, poderiam estar respondendo a esses movimentos com toques que teriam por objetivo cessar o movimento do feto, e não estabelecer um contato positivo com ele. Outra possibilidade seria a de que o próprio feto poderia estar apresentando um padrão motor mais acentuado e sensível em resposta ao estresse ou ansiedade da mãe.

Ao se depararem com achados controversos e inesperados, Hart e McMahon (2006) também reafirmaram a importância de considerar a complexidade desse fenômeno. Os autores não encontraram relações entre depressão e a preocupação da gestante com o feto, e ponderaram sobre o efeito de outras variáveis nesta relação como, por exemplo, o fato de a mãe ter outros filhos, de trabalhar fora em tempo integral ou ainda, de que problemas mais graves estivessem ocupando a gestante mais do que a gravidez. Para eles, os baixos escores de preocupação com o feto verificados, poderiam explicar a ausência de relações entre as duas variáveis investigadas.

No presente estudo, o número de filhos também se correlacionou negativamente com o apego materno-fetal e essa relação foi confirmada pela análise de regressão, embora a variância explicada tenha sido pequena. Os resultados da comparação entre o grupo de gestantes que estavam na primeira gravidez e o grupo que já havia engravidado antes também apoiam esta relação. Dos três estudos de revisão de literatura que foram examinados, Cannella (2005) abordou os efeitos desse fator demográfico específico sobre o apego maternofetal. Segundo a autora, tanto os estudos que correlacionam o número de filhos ao escore de apego materno-fetal quanto os que comparam gestantes primíparas e multíparas apontam para 
uma relação inversa entre as duas variáveis. As razões pelas quais isso acontece não foram suficientemente exploradas na literatura, mas uma hipótese possível seria a de que gestantes que não têm outros filhos teriam mais tempo livre e disponibilidade emocional para envolver-se com o feto.

A escolaridade materna e a escolaridade paterna também estiveram positivamente correlacionadas ao escore na escala de apego materno fetal, contudo, os valores foram baixos e essas relações não foram confirmadas pela análise de regressão. Essa mesma tendência já havia sido verificada por Lindgren (2001). Porém, os dois conjuntos de estudos revisados por Cannella (2005) e por Yarcheski et al. (2009) indicam que os achados da literatura a esse respeito são controversos, e que naqueles em que foram encontradas relações inversas entre a escolaridade e o apego materno-fetal, a força dessas relações foi pouco expressiva. De modo geral, as relações entre variáveis sociodemográficas e o apego materno-fetal costumam ser pouco consistentes, o que indica que elas não desempenham um papel fundamental. De qualquer modo, como esses dados costumam ser coletados na maioria das pesquisas, recomenda-se que elas continuem a ser investigadas, embora não devam constituir o principal foco de interesse nesta área (Yarcheski et al., 2009).

$\mathrm{O}$ presente estudo tem algumas limitações. Apesar do número expressivo de participantes, a técnica de amostragem utilizada não foi probabilística, o que pode representar uma limitação metodológica. Além disso, o instrumento utilizado para a avaliação do apego materno-fetal tem sido alvo de críticas da literatura (Doan et al., 2003), embora ainda seja a alternativa mais viável e promissora em se tratando de pesquisas que investigam amostras grandes. O mesmo pode ser apontado em relação ao instrumento utilizado para avaliar a saúde mental da gestante. Pelas suas características, o SRQ-20 não permitiu que o impacto de diferentes tipos de sintomas psiquiátricos (ex. depressão e ansiedade) fosse analisado separadamente. Além disso, se por um lado o SRQ-20 tem a vantagem de poder ser aplicado em larga escala, por outro, é um questionário de rastreamento, e não de diagnóstico. Nesse sentido, é possível que o uso de instrumentos mais precisos na detecção de transtornos como a ansiedade e a depressão possam resultar em um percentual maior da variância explicada no apego materno-fetal.

Em resumo, os achados do presente estudo corroboram a hipótese da literatura a respeito do impacto negativo dos sintomas psiquiátricos da gestante sobre o apego materno-fetal. Essas evidências apontam para a importância da avaliação e do acompanhamento psicológico durante o período pré-natal, para mulheres que apresentam esse tipo de sintomatologia. Contudo, por se tratar de uma área de pesquisa relativamente nova na Psicologia do Desenvolvimento, a respeito da qual pouco se sabe, recomenda-se cautela por parte das equipes de saúde mental nos processos de intervenção com base nos achados sobre o apego materno-fetal (Yarcheski et al., 2009). A complexidade das relações que se estabelecem entre inúmeros fatores durante a gestação e as constantes mudanças que ocorrem ao longo da mesma exigem uma abordagem sofisticada que considere as interações entre os diferentes fatores envolvidos. Assim, fica evidente a necessidade de que novas investigações ajudem a elucidar os mecanismos através dos quais diferentes tipos de sintomas psiquiátricos interferem no apego-materno fetal.

\section{Referências}

Alhusen, J. L. (2008). A Literature update on maternal-fetal attachment. Journal of Obstetric, Gynecologic, \& Neonatal Nursing, 37, 315-328.

Canella, B. L. (2005). Maternal-fetal attachment: an integrative review. Journal of Advanced Nursing, 50, 60-68.

Condon, J. T. (1985). The parental fetal-relationship - a comparision of male and female expectant parents. Journal of Psychosomatic Obstetrics and Gynecology, 4, 271-284.

Cranley, M. S. (1981). Development of a tool for measurement of maternal attachment during pregnancy. Nursing Research, 30, 281-284.

DiPietro, J. A. (2010). Psychological and psychophysiological considerations regardin the maternal-fetal relationship. Infant and Child Development, 19, 27-38.

Doan, H. M., Cox, N. L., \& Zimerman, A. (2003). The maternal fetal attachment scale: some methodological ponderings. Journal of Prenatal and Perinatal Psychology and Health, 18, 167-188.

Feijó, M. C. (1999). Validação brasileira da "maternal-fetal attachment scale"/ Brazilian validation of the maternal-fetal attachment scale. Arquivos Brasileiros de Psicologia, 51, 52-62.

Gonçalves, D. M., Stein, A. T., \& Kapczinski, F. (2008). Avaliação de desempenho do Self-Reporting Questionnaire como instrumento de rastreamento psiquiátrico: um estudo comparativo com o Structured Clinical Interview for DSM-IV-TR. Cadernos de Saúde Pública, 24, 380-390.

Hart, R., \& McMahon, C. A. (2006). Mood state and psychological adjustment to pregnancy. Archives Women's Ment Health, 9, 329-337.

Huang, H. C., Wang, S. Y., \& Chen, C. H. (2004). Body image, maternal-fetal attachment, and choice of infant feeding method: a Taiwan study. Birth, 31, 183-188.

Lindgren, K. (2001). Relationships among maternal-fetal attachment, prenatal depression, and health practices in pregnancy. Research in Nursing \& Health, 24, 203-217.

Mari, J., \& Williams, P. (1985). A comparison of the validity of two psychiatric screening questionnaires (GHQ-12 and SRQ-20) in Brazil, using Relative Operating Characteristic (ROC) analysis. Psychological Medicine, 15, 651-659.

Mari, J., \& Williams, P. (1986). A validity study of a psychiatric screening questionnaire (SRQ-20) in primary care in the city of São Paulo. British Journal of Psychiatry, 148, 23-26.

Robson, C. (1993). Real world research. Oxford: Blackwell.

Salisbury, A., Law K., LaGrase, L., \& Lester, B. (2003). Maternal-fetal attachment. Journal of the American Medical Association, 289(13), 1701. doi:10.1001/jama.289.13.1701

Seimyr, L., Sjögren, B., Welles-Nystrom, B., \& Nissen, E. (2009). Antenatal maternal depressive mood and parental-fetal attachment at the end of pregnancy. Archives Women's Ment Health, 12, 269-279.

Shieh, C., Kravitz, M., \& Wang, H. (2001). What do we know about maternal-fetal attachment. Kaohsiung Journal of Medical Sciences, 17, 448-454.

Shin, H., Park, Y-J., \& Kim, M. J. (2006). Predictors of maternal sensitivity during the early postpartum period. Issues and Innovations in Nursing Practice, 55, 425-434.

Yarcheski, A., Mahon, N. E., Yarcheski, T. J., Hanks, M. M., \& Cannella, B. L. (2009). A meta-analytuc study of predictors of maternal-fetal attachment. International Journal of Nursing Studies, 46, 708-715. 
Patrícia Alvarenga, doutora em Psicologia pela Universidade Federal do Rio Grande do Sul, é professora Adjunta da Universidade Federal da Bahia (UFBA). Endereço para correspondência: Universidade Federal da Bahia, Instituto de Psicologia, Rua: Aristides Novis, 197, Estrada de São Lázaro. CEP: 40210-730. Salvador, BA. E-mail: palvarenga66@gmail.com

Maria Virgínia Machado Dazzani, pós-doutora em Psicologia pela Clark University (USA), é professora Adjunta da Universidade Federal da Bahia (UFBA). E-mail: dazzani@superig.com.br

Cristiane Ajnamei dos Santos Alfaya, doutora em Psicologia pela Universidade Federal do Rio Grande do Sul, é professora Adjunta da Universidade Federal do Recôncavo da Bahia (UFRB).E-mail: c.alfaya@terra.com.br Eulina da Rocha Lordelo, pós-doutora pela University of Stavanger (Noruega), é professora Aposentada da Universidade Federal da Bahia (UFBA). E-mail: eulina@ufba.br

César Augusto Piccinini, pós-doutor pela University College London, é professor Associado da Universidade Federal do Rio Grande do Sul (UFRGS). E-mail: piccinini@portoweb.com.br 